\title{
A Strong Test of Exposure Effects
}

\author{
Richard L. Moreland and Robert B. Zajonc \\ University of Michigan
}

\begin{abstract}
A strong test of exposure effects was made by eliminating confounding demand characteristics through the use of a between-subject design. Each subject viewed novel stimuli at a single frequency level, and then rated them on several affective scales. Frequency of stimulus exposure was systematically varied across subjects. Stimuli were rated more positively with increasing exposure, despite the fact that subjects were unaware of any differences in exposure frequencies, and clearly unable to identify the experimental hypothesis. An awareness of the relationship between repeated exposure and affect was thus shown to be an unnecessary condition for the occurrence of exposure effects.
\end{abstract}

Over the past several years, many studies have investigated the hypothesis that the mere repeated exposure of a stimulus object is a sufficient condition for the enhancement of an individual's attraction toward that object. The hypothesized relationship has been obtained under a number of exposure conditions (Crandall, 1972; Harrison, 1969; Harrison \& Crandall, 1972; Zajonc, Crandall, Kail, \& Swap, 1974), and with a variety of stimulus objects (Heingartner \& Hall, 1974; Zajonc, 1968; Zajonc, Markus, \& Wilson, 1974). Recently, however, some questions have been raised about the validity of exposure effects: do the available data constitute evidence for a real psychological phenomenon, or are they simply the reflection of an experimental artifact? In particular, Stang (1974) has proposed that demand characteristics are a significant factor in the mere exposure effect.

Two approaches to the problem of demand characteristics may be found in the mere exposure literature. The first approach has relied upon the method of interpersonal replication (Bem, 1967). On the basis of an extensive investigation of this type, Stang (1974) has suggested that subjects in mere exposure experiments may be able to identify experimental hypotheses and produce corresponding patterns of results. In two role-playing experiments, for example, subjects were given questionnaires in which they were asked to imagine themselves as participants in an actual laboratory study. Subjects received detailed descriptions of both the stimuli and their frequencies of exposure, and gave affect ratings for each of

This work was supported by National Science Foundation Grant GS 35495. We thank Hazel Markus and Bill Wilson for reading an earlier version of the manuscript. Requests for reprints should be sent to Richard L. Moreland, Institute for Social Research, University of Michigan, Ann Arbor, Michigan 48106. 
the hypothetical stimuli. These affect ratings were found to vary with the described exposure frequencics in a manner that was similar to the results of several laboratory studies (Harrison \& Hines, 1970; Janisse, 1970; Zajonc \& Rajecki, 1969). The subjects were also asked to make guesses about a probable experimental hypothesis; more than a third of them mentioned some relationship between affect ratings and frequency of exposure, and the majority of those responses involved a positive association.

In two other experiments, Stang (1974) actually showed stimuli to subjects in variable exposure frequencies. The subjects were told before the exposure series that stimulus affect ratings would be obtained, and an explicit mention of differences in exposure frequencies was also made. After making the stimulus affect ratings, the subjects were asked about the hypothesis which they believed the experimenter was testing. A questionnaire was distributed in which five hypotheses were listed, three of them involving some relationship between exposure and affect. More than $60 \%$ of the subjects in both experiments selected one of those three hypotheses, and many of those selections indicated a positive relationship to be the most likely possibility. ${ }^{1}$ Affect ratings were again found to vary positively with frequency of exposure, and a close association between affect rating patterns and hypothesis selections was revealed. Apparently, an awareness of some relationship between stimulus exposure and affect was an important factor in all of these experiments.

A second approach to the problem of demand characteristics has involved the use of procedures designed to conceal experimental hypotheses from the subjects. Some studies of this type have been carried out in which subjects' views about the purpose of thcir research participation were probably incorrect, and yet a positive relationship between exposure and affect was still found (Rajecki \& Wolfson, 1973; Saegert, Swap, \& Zajonc, 1973). In the Saegert, Swap, and Zajonc (1973) study, for example, subjects were led to believe that they were taking part in research on the psychophysics of taste. Actually, the frequency of encounters between the subjects was unobtrusively varied, and measures of interpersonal liking were obtained through the use of items imbedded in a "general questionnaire" dealing with other details of the experiment. Interpersonal liking was found to vary positively with frequency of encounter; however, systematic data regarding subjects' guesses about experimental hypotheses were not collected in all of the experiments. While none of the subjects in Experiment II mentioned interpersonal attraction as a possible experimental hypothesis, the extent to which the subjects in Experiment I were aware of research interests was not

${ }^{1}$ This percentage was calculated directly from data supplied by David J. Stang on September 24, 1974. 
determined. The success with which hypotheses in the Rajecki and Wolfson (1973) experiments were concealed is also not known.

Zajonc, Swap, Harrison, and Roberts (1971) attempted to conceal experimental hypotheses through the use of a between-subject design. In one part of that study (Experiment III), the subjects viewed stimuli under conditions in which the correct identification of research interests was made nearly impossible. Frequency of exposure was varied between subjects, i.e., each subject viewed stimuli at a single frequency level, and was presumably unaware that his responses might be compared with those of other subjects viewing stimuli at other frequency levels. No clear relationship between exposure and affect was found, although the data displayed a trend suggesting a positive association between the two variables. In another part of the study (Experiment IV), variations in the frequency of exposure to four classes of stimuli (trees, flowers, fruits, and vegetables) were based upon previously established word counts (Thorndike \& Lorge, 1944). Each group of subjects rated stimuli at a different frequency level, and a significant exposure effect was obtained. These results cannot, however, be taken as unequivocal proof against the operation of demand characteristics artifact. For some groups of subjects, especially those asked to give affect ratings to uncommon stimuli, frequency of exposure may have been a conspicuous stimulus attribute, allowing for correct inferences about the experimental hypotheses.

The literature on demand characteristics in mere exposure research presents a rather ambiguous pattern of results, and yet the methodological approaches contained in that literature are still quite valid and useful. Interpersonal replications, for example, can provide interesting information about the reactions of subjects to particular research conditions. A strong test of exposure effects, however, requires that experimental hypotheses be clearly and successfully concealed from the subjects. The following experiment provided such a test by utilizing a between-subject design in an extension of the Zajonc, Swap, Harrison, and Roberts (1971) research. Several modifications in both the procedure and analysis of that research were made to allow for an unambiguous evaluation of exposure effects. The experiment thus attempted to discover if a positive frequency-affect relationship could be obtained even under conditions in which a demand characteristics explanation was untenable.

\section{METHOD}

Subjects. Subjects were 90 undergraduate women at the University of Michigan, none of whom had taken part in similar research. Participation in the experiment served to fulfill a course requirement.

Materials. Twenty-seven $35-\mathrm{mm}$ slides of Japanese ideographs were used as stimuli. The slides were projected by means of a Keystone 1055 projector, and exposure timing was controlled through the use of a Lafayette 43011-16 electronic shutter.

Procedure. Subjects werc randomly assigned to one of six groups, each containing 15 members. These groups represented particular combinations of three factors: the number of 
different slides seen, their frequency of exposure, and the length (total number of exposures) of the exposure sequence. The multiplicative relationship between these factors precluded the use of a complete factorial design; specific factor levels were instead chosen in such a way that the maximum amount of experimental information was gained. A summary of the factor levels for each of the six groups is shown in Table 1. Three of the groups saw only three different slides, one group viewing slides three times apiece, another group viewing slides nine times apiece, and a third group viewing slides 27 times apiece. Two other groups saw nine different slides, one group viewing slides three times apiece, and the other group viewing slides nine times apiece. The remaining group saw 27 different slides three times apiece. Exposure sequences thus varied from nine to 81 slides in length. Three slides were common to all of the groups, and were used for rating purposes later in the experiment.

Each experimental session involved three subjects drawn from one of the six treatment groups, but no communication between subjects was allowed during the course of the experiment. Upon entering the laboratory, the subjects were asked to watch a series of slides. No other instructions were given at that time.

Each stimulus was shown for $2 \mathrm{sec}$, with a 5 -sec interval between each presentation. Order of presentation was randomized separately for each of the six groups. After all slides had been shown, a set of seven-point semantic rating scales was given to each subject. LIKE-DISLIKE, GOOD-BAD, and PLEASANT-UNPLEASANT ratings were then obtained for each of three different stimuli. Scale endpoints and orders were rotated across subjects. All subjects rated only three stimuli, the same for all six groups, and they were told to work quickly and to indicatc only their first impressions. During the rating phase, each stimulus was again shown for $2 \mathrm{sec}$. Twenty seconds were allowed for the completion of the three ratings for each stimulus.

After the affect ratings had been collected, the subjects were asked to make guesses about possible experimental hypotheses or research interests. Finally, the subjects were told to think back on their participation in the experiment, and to concentrate on their thoughts and emotions during the exposure sequence. As a global measure of experimental satisfaction, the subjects were then asked to rate the experience of watching the slides on a seven-point PLEASANT-UNPLEASANT scale. Those ratings were collected, and the subjects were debriefed, allowed to ask questions, and dismissed.

\section{RESULTS}

The affect ratings for each subject were averaged over scales and stimuli to produce a composite affect score. A summary of the affect scores and experimental satisfaction ratings for each of the six groups is shown in Table 1.

The nature of the experimental design required that the data be analyzed in a multiple regression format (Cohen, 1968; Draper \& Smith, 1966; Wolf \& Cartwright, 1974). Under this procedure, a number of "dummy" variables were created to account for particular degrees of freedom in the design. Linear and quadratic effects for both stimulus diversity and frequency of exposure were treated in this manner. In order to preserve a nonsingular data matrix, only two levels of stimulus sequence length were coded; an assumption of equal effects in the two shorter sequences (nine and 27) was made, and only a long-short dichotomy was preserved. All of the "dummy" variables were first coded on a $-1,0,+1$ basis, and then adjusted for zero means. 
TABLE 1

AFFECT SCORES AND EXPERIMENTAL SATISFACTION RATINGS ${ }^{a}$

\begin{tabular}{lrrrrrrr}
\hline & \multicolumn{7}{c}{ Group number } \\
\cline { 2 - 8 } \multicolumn{1}{c}{ Group characteristic } & 1 & 2 & 3 & 4 & 5 & 6 \\
\hline Number of different stimuli & 3 & 9 & 27 & 3 & 9 & 3 \\
Frequency of stimulus exposure & 3 & 3 & 3 & 9 & 9 & 27 \\
Length of exposure sequence & 9 & 27 & 81 & 27 & 81 & 81 \\
Affect scores & & & & & & \\
$\quad$ Mean & 3.39 & 3.44 & 3.39 & 3.78 & 3.59 & 4.07 \\
$\quad$ Standard deviation & 0.53 & 0.64 & 0.57 & 0.46 & 0.51 & 0.61 \\
Experimental satisfaction ratings & & & & & & \\
$\quad$ Mean & 3.87 & 3.47 & 2.93 & 3.67 & 3.07 & 3.13 \\
$\quad$ Standard deviation & 0.99 & 0.99 & 1.03 & 0.90 & 0.96 & 1.06 \\
\hline
\end{tabular}

${ }^{a}$ Both affect scores and experimental satisfaction ratings become more positive with increasing magnitude.

In the first regression analysis, the "dummy" variables were used to predict the affect scores. This regression proved to be significant, $F(5,84)=3.58, p<.01$, but only the linear component for frequency of exposure showed a significant partial correlation with affect, $r(84)=.27$, $p<.03$. The multiple correlation between the predictor variables and the affect scores was .42 , which yielded an $R^{2}$ of .18 . The combined average affect rating for three exposures was 3.41 , for nine exposures 3.68 , and for 27 exposures 4.07 . The linear slope generated by these affect ratings against the logarithm of frequency of exposure was .78, remarkably similar to the slopes found in other exposure studies (e.g., Zajonc, 1968; Zajonc, Markus, \& Wilson, 1974).

In a second regression analysis, the "dummy" variables and the experimental satisfaction ratings were used to predict the affect scores. This regression was also significant, $F(6,83)=4.46, p<.001$. The multiple correlation between the predictor variables and the affect scores was .49, yielding an $R^{2}$ of .24. Significant partial correlations with affect were found for both the linear component of frequency of exposure, $r(83)=.27$, $p<.02$, and the ratings of experimental satisfaction, $r(83)=.29, p<.01$. Apparently, both factors made important and independent contributions to the determination of affect scores. The nature of the relationship between experimental satisfaction and affect is of some interest, in that other studies (e.g., Saegert, Swap, \& Zajonc, 1973; Zajonc, Markus, \& Wilson, 1974) have shown context effects to be associated primarily with the intercept, rather than the slope, of the linear function relating exposure and affect. This type of relationship was also found in the data reported here. Subjects were divided according to their position above or below the median 
experimental satisfaction rating. The linear slopes (affect ratings against the logarithm of frequency of exposure) for the two groups were almost identical: the slope for subjects above the median was .79, while the slope for subjects below the median was .76. The overall elevation (average affect score) of the two groups. however, was significantly different, $t(88)=1.77, p<.05$, supporting the earlier experimental findings.

The hypotheses guessed by the subjects were examined independently by three judges, using a simple categorization scheme developed in earlier research. Final response categorizations were made on the basis of their decisions. The judges were found to be in agreement on about $90 \%$ of these responses. More than one-third of the subjects were unable to make any guesses at all about the purpose of the experiment. Less than $5 \%$ of the subjects proposed any relationship between exposure and affect, and only two individuals mentioned a positive frequency-affect relationship as a possible hypothesis.

\section{DISCUSSION}

The results revealed that a demand characteristics artifact does not underlie the relationship between stimulus exposure and affect. A typical exposure effect was obtained despite the fact that subjects only viewed stimuli at a single frequency level, and thus could not have based their affect ratings upon either comparisons with stimuli viewed at any other frequency levels, or upon experimental interpretations involving any sort of frequency considerations. Apparently, an experimental demonstration of the frequency-affect relationship does not depend upon subjects' recognition of research interests. In addition, the data did not support the proposition that exposure context is the only determining factor in the mere exposure effect (Burgess \& Sales, 1971; Suedfeld, Epstein, Buchanan, \& Landon, 1971). Frequency of exposure was found to be associated with the slope, and experimental satisfaction with the intercept, of a linear function relating exposure and affect, and a significant positive correlation between exposure and affect was found even when the effects of experimental satisfaction were held constant.

An integration of these results into the existing literature on demand characteristics and mere exposure research raises two important issues. First, some explanation must be found for the lack of significant exposure effects in the similar study performed by Zajonc, Swap, Harrison, and Roberts (1971). Those authors attributed the nature of their data to the vagaries of rating scale behavior; subjects distributed their responses to the different stimuli over a wide range on the rating scales, generating substantial variance within each frequency level. The exposure effect may also have been attenuated by the fact that neither the number of different stimuli seen by each subject, nor the ratio of familiar to unfamiliar stimuli contained within the rating phase, were treated as systematic sources of 
variation in affect ratings. Naturally, these explanations are somewhat speculative, and may have little to do with the actual determination of affect ratings. Nevertheless, the pattern of results in that study was similar to that reported here, although the exposure effect was much weaker (the linear slope ${ }^{2}$ of affect ratings against the logarithm of frequency of exposure was only .14). It seems reasonable to assume, therefore, that the procedural and analytical modifications made in the present study were sufficiently extensive to allow for a clearer operation of exposure effects.

A second issue involves the actual prevalence of demand characteristics in mere exposure research. Although the results reported here have shown that a demand characteristics artifact does not underlie the frequencyaffect relationship, it is still possible that demand characteristics play an important role in the majority of exposure studies. The results reported by Stang (1974) have dramatized that possibility, but may be misleading with regard to the operation of demand characteristics under more typical research conditions. Demand characteristics are determined by the ways in which subjects interpret their experimental participation, and those interpretations are themselves dependent upon both the type and the amount of information that is available during the course of the experiment. In mere exposure research, interpretations involving the frequency-affect relationship depend primarily upon the availability of information regarding differences in exposure frequencies. The mere availability of frequency information, though, is not a sufficient condition for the elicitation of confounding demand characteristics. The degree to which subjects are aware of differences in exposure frequencies, and the significance which they attach to that information, are also of some importance.

In general, demand characteristics may produce an artifactual exposure effect if (a) differences in exposure frequencies are made salient to the subjects; (b) an experimental interest in stimulus affect ratings is conspicuous; and (c) subjects identify a positive frequency-affect relationship as the experimental hypothesis and deliberately rate the stimuli in a manner reflecting that relationship. In a typical research situation, the subjects are probably only vaguely aware of differences in exposure frequencies, and may regard such differences as relatively unimportant in comparison to any perceived characteristics of the stimuli or their style of presentation. At the time of exposure, the subjects do not know that they will be required to give an affect rating to each stimulus, and any subsequent discriminations of stimuli along a frequency dimension must depend upon the accuracy of frequency recall. Also, any inferences about research interests must be made by the subjects themselves, without any aid from the experimenter.

2 This slope was calculated from data produced by subjects who were in the control condition, and for whom stimuli were counterbalanced across frequency levels. 
These typical research conditions may be contrasted with those created and used by Stang (1974). Subjects in his two role-playing studies were given information about the frequency of stimulus exposure that was both specific and salient, and no other information about the research setting was made available at any time. In the two other studies, subjects were provided with several alternative hypotheses by the experimenter, rather than generating their own on the basis of their research experience. Information about exposure frequencies was also given to the subjects before any of the stimuli were actually seen. In fact, all of the Stang (1974) experiments involved some features that may have led to a heightened awareness of the frequency-affect relationship on the part of the subjects, and the results of those experiments should probably not be taken as evidence for the operation of a demand characteristics artifact in mere exposure research.

Interpersonal replication studies can provide valuable information about demand characteristics if the experimental participation of actual laboratory subjects is accurately simulated. For that reason, two experiments were performed which examined the problem of demand characteristics by focusing on the availability, specificity, and salience of frequency information. ${ }^{3}$ These experiments made use of interpersonal replication methods similar to those of Stang (1974), but an attempt was made to adjust the information given to subjects in ways that more faithfully reflected a true laboratory situation. It was thus possible to re-examine the degree to which subjects are able to accurately identify experimental hypotheses under simulated research conditions. In the first experiment, information about differences in exposure frequencies was given to the subjects and made salient. These conditions enabled about $45 \%$ of the subjects to propose hypotheses involving some association between exposure and affect; however, two-thirds of those hypotheses failed to describe the direction that such an association might take. In the second experiment, the salience of frequency information was reduced, and almost no subjects suggested that repeated stimulus exposure might enhance affect ratings. Few subjects in either experiment proposed a positive frequency-affect relationship as an experimental hypothesis; in fact, many more professed to have had no ideas at all about the purpose or meaning of the described research. These results strongly suggest that an awareness of the relationship between exposure and affect is relatively rare among subjects participating in typical mere exposure research.

In conclusion, it seems that the role of demand characteristics in mere exposure research has been misunderstood in several ways. Stang's (1974) conclusions on the importance of demand characteristics were based upon data generated by subjects for whom relevant experimental information had been provided that was unusually specific and salient. When the

\footnotetext{
${ }^{3}$ A more detailed description of these two experiments may be obtained from the authors.
} 
information given to subjects is adjusted to levels that more nearly approximate those of actual laboratory conditions, however, the prevalence of a demand characteristics artifact becomes somewhat questionable. Furthermore, even the limited operation of such an artifact is unnecessary for the occurrence of the mere exposure phenomenon. A significant exposure effect is obtained even when subjects are unaware of differences in exposure frequencies, and unable to identify experimental hypotheses. Clearly, the mere repeated exposure of a stimulus object is a sufficient condition for the enhancement of an individual's attraction toward it.

\section{REFERENCES}

Bem, D. J. Self-description: An alternative interpretation of cognitive dissonance phenomena. Psychological Review, 1967, 74, 183-200.

Burgess, T. D. C., \& Sales, S. M. Attitudinal effects of "mere exposure": A reevaluation. Journal of Experimental Social Psychology, 1971, 7, 461-472.

Cohen, J. Multiple regression as a general data-analytic system. Psychological Bulletin, $1968,70,426-443$.

Crandall, R. Field extension of the frequency-affect findings. Psychological Reports, 1972 , 31, 371-374.

Draper, N., \& Smith, H. Applied regression analysis. New York: Wiley, 1967.

Harrison, A. A. Exposure and popularity. Journal of Personality, 1969, 37, 359-377.

Harrison, A. A., \& Crandall, R. Heterogeneity-homogeneity of exposure sequence and the attitudinal effects of exposure. Journal of Personality and Social Psychology, 1972, 21, 234-238.

Heingartner, A., \& Hall, J. V. Affective consequences in adults and children of repeated exposure to auditory stimuli. Journal of Personality and Social Psychology, 1974, 29, $719-723$.

Janisse, M. P. Attitudinal effects of mere exposure: A replication and extension. Psychonomic Science, 1970, 19, 77-78.

Rajecki, D. W., \& Wolfson, C. The rating of materials found in the mailbox: Effects of frequency of receipt. Public Opinion Quarterly, 1973, 37, 110-114.

Saegert, S., Swap, W., \& Zajonc, R. B. Exposure, context, and interpersonal attraction. Journal of Personality and Social Psychology, 1973, 25, 234-242.

Stang, D. J. Intuition as artifact in mere exposure studies. Journal of Personality and Social Psychology, 1974, 30, 647-653.

Suedfeld, P., Epstein, Y. M., Buchanan, E., \& Landon, P. B. Effects of set on the "effects of mere exposure." Journal of Personality and Social Psychology, 1971, 17, 121-123.

Thorndike, E. L., \& Lorge, I. The teacher's wordbook of 30,000 words. New York: Teacher's College, Columbia University, 1944.

Wolf, G., \& Cartwright, B. Rules for coding dummy variables in multiple regression. Psychological Bulletin, 1974, 81, 173-179.

Zajonc, R. B. Attitudinal effects of mere exposure. Journal of Personality and Social Psychology, 1968, 9, 1-27.

Zajonc, R. B., Crandall. R., Kail, R. V., \& Swap, W. Effects of extreme exposure frequencies on different affective ratings of stimuli. Perceptual and Motor Skills, 1974, $38,667-678$. 
Zajonc, R. B., Markus, H., \& Wilson, W. R. Exposure effects and associative learning. Journal of Experimental Social Psychology, 1974, 10, 248-263.

Zajonc, R. B., \& Rajecki, D. W. Exposure and affect: A field experiment. Psychonomic Science, 1969, 17, 216-217.

Zajonc, R. B., Swap, W., Harrison, A. A., \& Roberts, P. R. Limiting conditions of the exposure effect: Satiation and relativity. Journal of Personality and Social Psychology, 1971, 18, 384-391.

(Received November 28, 1974) 